\title{
Design and measurement of stress indicator structures for the characterization of Epoclad negative photoresist
}

\author{
Kristof Wouters ${ }^{1}$ and Robert Puers \\ Department Electrotechniek ESAT-MICAS, K.U.Leuven, Kasteelpark Arenberg 10, B-3001 Leuven, \\ Belgium \\ E-mail: Kristof.wouters@esat.kuleuven.be
}

Received 18 December 2008, in final form 30 March 2009

Published 30 June 2009

Online at stacks.iop.org/JMM/19/074019

\begin{abstract}
Using new materials for the structural or active layers in MEMS requires the knowledge of the material properties. In this paper, the internal stress and the coefficient of thermal expansion of Epoclad negative photoresist are measured. Despite being a photoresist, the epoxy-based material has very good mechanical properties. It enables the creation of microscale mechanical structures using this material. The internal stress and the coefficient of thermal expansion were measured using on-wafer stress indicator structures. The structures were designed to give a response caused by internal stress measurable using an optical microscope. The coefficient of thermal expansion is measured via the internal stress at different temperatures.
\end{abstract}

\section{Introduction}

Historically, the fabrication of MEMS uses technologies to micromachined silicon or other semiconductor-based materials. However, in the past decade other materials have also been introduced and used for the active or structural parts of MEMS [1-3]. The goal is to make cheaper or even new and better MEMS using these new materials. Among these new materials, some polymers have good mechanical properties. There are many different polymer materials but not all are suitable for MEMS. Despite this, the useful polymers provide a broad spectrum of material properties, not only mechanically but also chemically and electrically.

To obtain polymer-based microscaled moving structures, it is good to start with a material that promises good mechanical stability. Thus, a thermoset polymer is a good choice. These types of polymers will significantly deform or melt only at very high temperatures. This is in contrast with thermoplastic materials, which can easily melt at relative low temperatures. Examples of such thermoplastics are the typical polymers in our everyday use. They can easily be molded using, for

\footnotetext{
1 Author to whom any correspondence should be addressed.
}

instance, injection molding to make all kinds of shapes for packing material.

The material being characterized here is an epoxy. This means that the polymer network is highly crosslinked. It is this crosslinked network that gives the epoxy its stability, both chemically and mechanically, and it is classified as a thermoset. The Epoclad negative resist is a photocurable epoxy. The material is spin coatable and after a soft bake step to evaporate the solvent the film is exposed to UV light. The material is then cured in an oven. Uncured parts of the film can be redissolved during a development step. Thus, the material can be patterned using the conventional lithographic equipment. In addition, the Epoclad epoxy also has good adhesion to various substrates, including silicon wafers. All these properties make Epoclad a good material to fabricate microscale mechanical structures.

The Epoclad is an insulator which is not ideal for a MEMS material. Thus, in order to render it conductive different methods can be used. The material can be blended with a conductive filler. For instance, silver particles, carbon nanotubes or conductive polymers can be used. Alternatively, the cured epoxy structure can be coated with a metal layer; see figure 1 . 


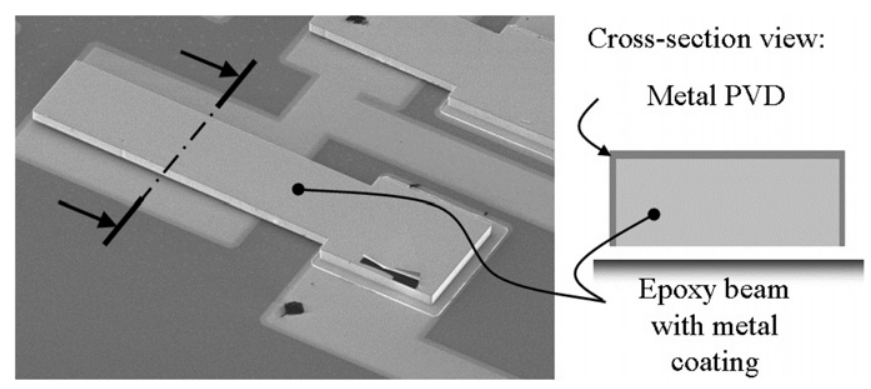

Figure 1. An example of an in situ fabricated epoxy beam coated with a sputtered (physical vapor deposition) metal layer. The beam is a free-standing SU-8 structure with a gap of $3 \mu \mathrm{m}$ and a length of $400 \mu \mathrm{m}$.

SU-8 is a very analog negative resist compared to Epoclad. It is also a photoepoxy and has good mechanical and chemical properties, like Epoclad. SU-8 has been very well reported in the literature so far. Here, the material is tested in the same way as Epoclad for comparison. To the authors' knowledge, the internal stress and the coefficient of thermal expansion of Epoclad have not been reported yet.

To be complete and to further introduce the Epoclad negative resist, Epoclad originated in the manufacturing of integrated optical waveguides. Epoclad comes together with Epocore, also a negative epoxy-based resist. To manufacture an optical waveguide, Epocore would be used as the core and Epoclad as the cladding of the waveguide [3]. Epocore, however, has very bad adhesion properties to various substrates such as silicon wafers. For this reason, it is not very well suited to be used separately from Epoclad to make micro mechanical structures, whereas Epoclad can be used separately from Epocore as is done in this study.

The internal stress and the coefficient of thermal expansion (CTE) together with the Young's modulus are the first parameters that one needs to make a finite element simulation of the mechanical behavior. This paper deals with the first two parameters. Many different approaches have been used in order to measure the built-in stress and the CTE; for instance, measuring the curvature of a wafer coated with the material of interest or measuring the force versus the displacement of a membrane made of the material of interest [4-9]. The recorded data are then typically fitted to an analytical model to determine properties such as the Young's modulus and the internal stress. These types of experiments require equipment to accurately measure displacements or applied forces. When using on-wafer stress indicator structures, the experiment is more straightforward.

Stress indicator structures are MEM structures that will produce a displacement that is, in this case, measurable using an optical microscope. So, right after the fabrication process the MEM structures can be measured and the internal stress recorded.

The outline of this paper is as follows. The following section focuses on the indictor structure itself. The details of the design are given: how the design is scaled in order to cope (at the design stage) with the unknown material parameters. Next, an overview of the fabrication process is given. The

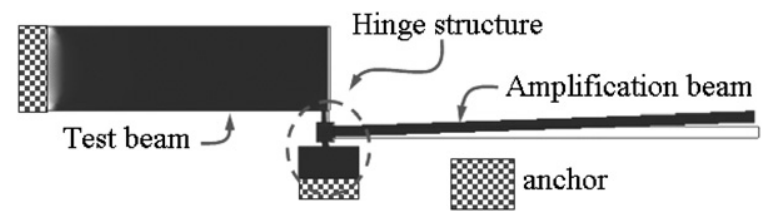

Figure 2. The relative ridged test beam will produce a strain caused by the internal stress once the sacrificial layer is etched away. A hinge structure and a long amplification beam amplify this strain into an optical visible displacement.

final two sections deal with the measurement results and discussion.

\section{Design of the indicator structures}

The working principle of the MEM indicator structure is straightforward; see figure 2 . The basic idea is to fabricate a free-standing structure with the material of interest. This is typically done using a sacrificial layer underneath the structure. When the sacrificial layer is etched away, the free-standing beam can move or deform. When there is originally a tensile stress in the structure, the beams will shrink. This usually small displacement is amplified using some kind of hinge structure. In this way, the recorded displacement can be measured with higher accuracy and this can be done using a conventional microscope.

Different designs have been proposed in the past decades for these on-wafer stress indicator structures. Gianchandani and Najafi [6] proposed a design using double-clamped cantilevers. This is useful when the deposited material has a large stress gradient in the out-of-plane direction. This will cause the beams to bend upward or downward. However, when stress gradients are low, no major out-of-plane bending will occur. In this case, no double-clamped beams are needed [9].

While designing our stress indicator structures, three major points need attention. (i) First, we assume that SU8 is a very analog material to Epoclad. For SU-8 the built-in stress at room temperature is very high relative to the fraction limit of the material [11-13]. This means that we have to make the structures safe enough so that they do not break. In other words, we have to ensure that no stress accumulation occurs in the structure. (ii) Second, the modeling in a finite element simulator is complicated by the fact that we do not know the built-in stress in advance. (iii) Finally, the on-wafer structures need to produce a displacement large enough so that the relative error on the measurement is reduced. In the rest of this section, these three points will be discussed further.

(i) At the design phase, the built-in stress was not known for Epoclad. The Young's modulus, however, was already measured in a previous study [12]. Figure 3(a) shows the stress-strain plot of Epoclad. The material behaves very linearly up to $1 \%$ strain. This $1 \%$ strain will be set as the maximum allowed strain in the structure in order to ensure that critical parts of the stress indicator structure do not fail. To illustrate further, the hinge structure is a very critical part regarding high stresses and strains. A hinge will typically 


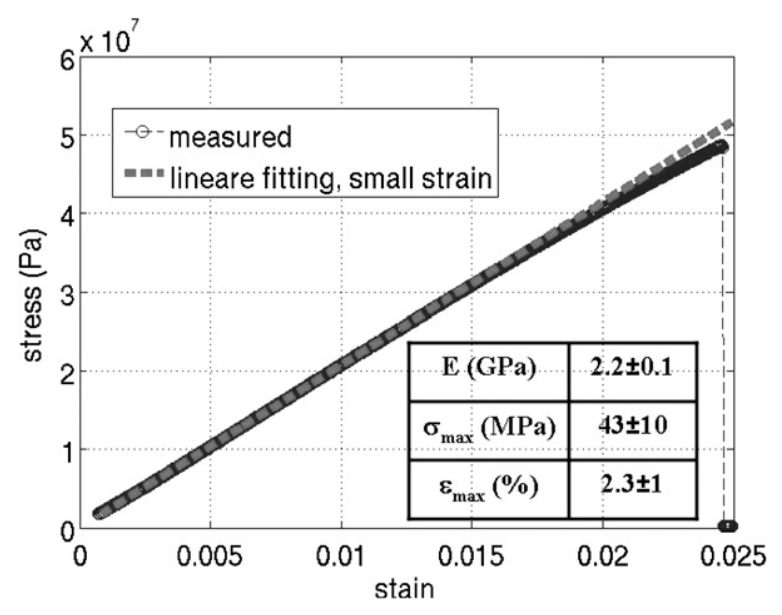

Figure 3. Stress-strain curve of Epoclad. A $5 \mathrm{~mm}$ by $20 \mathrm{~mm}$ and $60 \mu \mathrm{m}$ thick Epoclad film was stretched at $3 \mu \mathrm{m} \mathrm{s}^{-1}$ until fracture. The Young's modulus, the stress and strain upon fracture $\left(\sigma_{\max }\right.$, $\left.\varepsilon_{\max }\right)$ are averaged over approximately 40 samples. The linear fit uses the stress-strain data from the lowest data point up to 0.005 strain $(0.5 \%$ strain $)$

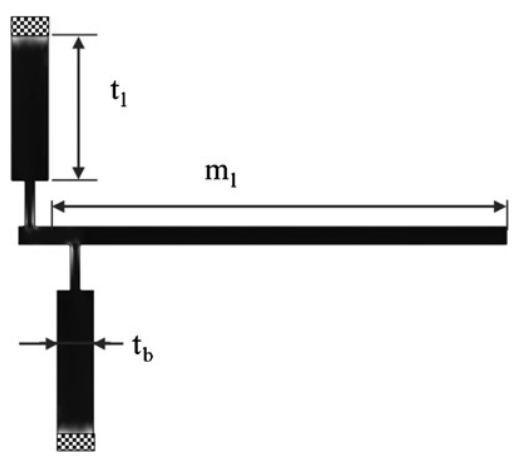

Figure 4. Chosen design for the stress indicator structures proposed by Goosen et al [9]. The test beam length $\left(t_{1}\right)$ and the test beam width $\left(t_{b}\right)$ are the major parameters that have to be determined.

feel a stretching force and a bending momentum. Around the hinge anchor points, stress concentration can also lead to very high stresses. For example, implementing a stress indicator structure as in figure 2 and assuming that the material is in tensile stress, the hinge structure will stretch and bend. This extra stress will be superimposed on the original built-in stress. Thus, when the structure is released and the sacrificial layer is etched away, the net stress in the hinge will be even higher than the built-in stress of the material.

In other words, a design wherein such stress accumulation occurs must be avoided. A design proposed by Goosen et al [9] ensures that the hinges do not suffer from stresses that are superimposed on to the initial built-in stress; see figure 4. This is because the hinges - the most critical part for the stress accumulation-are only loaded with a bending force and not with a tensile force. This allows the hinges to relax most of their built-in stress.

Because the stress gradient in the Epoclad epoxy is low enough, it will not produce a major bending up or down. The long single-clamped beam, the amplification beam, does not pose a problem.
Table 1. Sizing of the three different indicator structures. The resolution is the minimal recordable stress.

\begin{tabular}{lclll}
\hline Device & $\begin{array}{l}\text { Test beam } \\
\text { length }(\mu \mathrm{m})\end{array}$ & $\begin{array}{l}\text { AB length } \\
(\mu \mathrm{m})\end{array}$ & $\begin{array}{l}\text { Range } \\
(\mathrm{MPa})\end{array}$ & $\begin{array}{l}\text { Resolution } \\
(\mathrm{MPa})\end{array}$ \\
\hline Device 1 & 100 & 1000 & $15-20$ & 1 \\
Device 2 & 250 & 1000 & $2-15$ & 0.7 \\
Device 3 & 2000 & 1500 & $0.2-2$ & 0.07 \\
\hline
\end{tabular}

(ii) Now we know that the design proposed by Goosen et al is well suited for a stress indicator structure made of Epoclad. The design can cope with the fact that the epoxy has very high internal stresses relative to the fracture limit of the material. But, the built-in stress is unknown. Depending on the built-in stress, the dimensions of the stress indicator structure have an optimum. When we increase the length of the test beam, the displacement of the amplification beam will be larger, and also the bending inside the hinges. For this reason, three different sizes of the stress indicator structures will be implemented by altering the dimensions, each having an optimal operating range of built-in stresses; see table 1 .

To determine the optimal sizes of the indicator structure a detailed finite element simulation was done. The Comsol Multiphysics software was used as the core solver for the simulations. In order to simplify the sizing problem, the hinge dimensions were arbitrary chosen to be $20 \mu \mathrm{m}$ by $80 \mu \mathrm{m}$. This leaves the dimensions of the test beam as the to-be-determined parameters. In one simulation loop, the built-in stress is swept through a specific range for a set of fixed dimensions of the test beam. In the next loop, the test beam's width $\left(t_{b}\right)$ and the test beam's length $\left(t_{1}\right)$ are changed and again the stresses and responses are calculated in the structure; see figure $5(\mathrm{~g})$. In figures $5(a)-(f)$ an example of such a simulation output is shown. This is the output of device 2. It is clear that the test beam length $\left(t_{1}\right)$ has a major influence on the response of the indicator structure. The test beam width $\left(t_{\mathrm{b}}\right)$ has a negligible influence. But for the maximum stresses and strains in the hinges, the test beam width is significant.

(iii) Because the stress indicator structure needs as large response as possible, the test beam length must be as large as possible so that the maximum strain stays below $1 \%$. The test beam width must also be as large as possible because the test beam must be ridged enough. A thinner test beam will reduce the stress inside the hinges, but will also lead to more fragile structures.

To conclude the sizing problem, three devices were sized using the design proposed by Goosen et al. The stress indicator structures cover a range of $0.2-20 \mathrm{MPa}$ of built-in stress and they can measure both compressive and tensile stresses. In the final implementation, two of the same structures are placed adjacent to each other. The amplification beams face each other and they are point mirror images of each other; see figure 6 . In this way, the two adjacent structures will produce a displacement in the opposite direction. This will further reduce the measurement error by a factor of 2 . A vernier grating is placed on the amplification beam apex to help with the measurements.

The same structures can also be used to determine the CTE. This is because Epoclad is an epoxy material and thus 

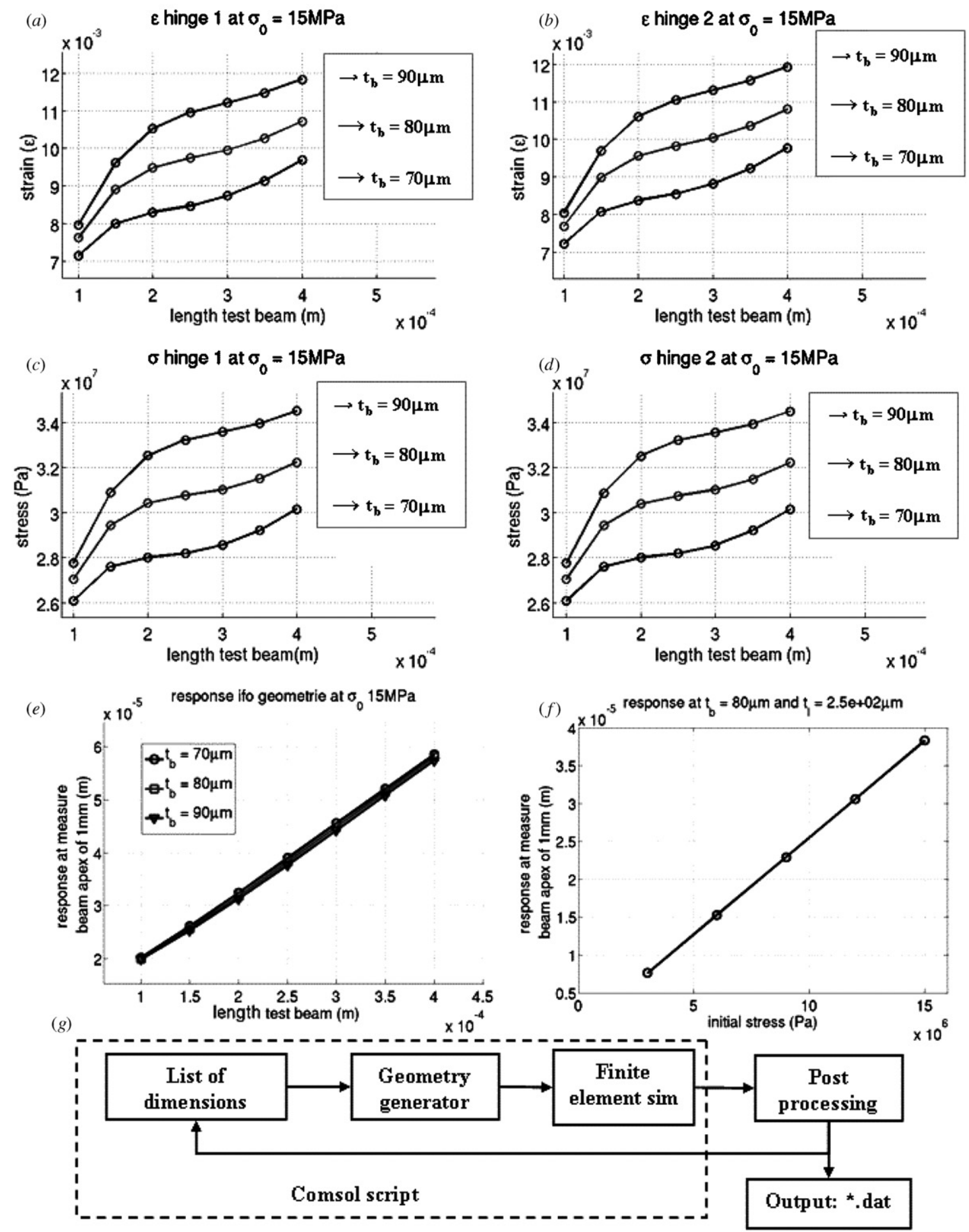

Figure 5. $(a)$ and $(b)$ give the maximum strain inside the hinges as a function of the test beam length and width. $(c)$ and $(d)$ give the maximum stress inside the hinges as a function of the test beam length and width. $(e)$ gives the response of the stress indicator structure as a function of the test beam length and test beam width. $(f)$ displays the response as a function of the internal stress for a fixed test beam length and width. $(g)$ Simulation diagram.

the CTE is expected to be large and comparable to that of SU-8. Finite element simulation reveals that the indicator structures produce a displacement of the same order of magnitude as the internal stress at moderate temperature increases; up to $150{ }^{\circ} \mathrm{C}$ is sufficient. To quantify 'in the same order of magnitude' one can simply calculate the temperature step needed to cancel out a displacement caused by internal tensile stress. This is as follows:

$$
\Delta T=\frac{\sigma_{0}}{E \cdot \alpha} .
$$

Here $\sigma_{0}$ is the internal stress, $E$ is the Young's modulus and $\alpha$ is the CTE. For typical values of silicon, the required temperature 


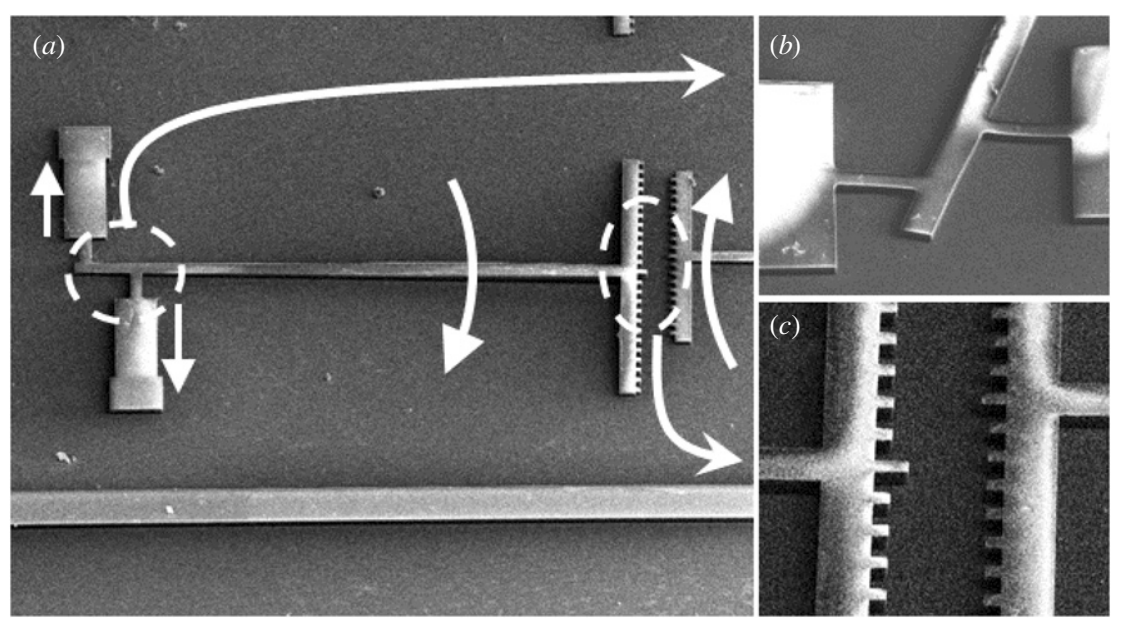

Figure 6. (a) Fabricated indicator structure showing a displacement caused by tensile stress. (b) Detail of the very flexible hinges. The adjacent structure is the point mirror image of the first so there the displacement is opposite.

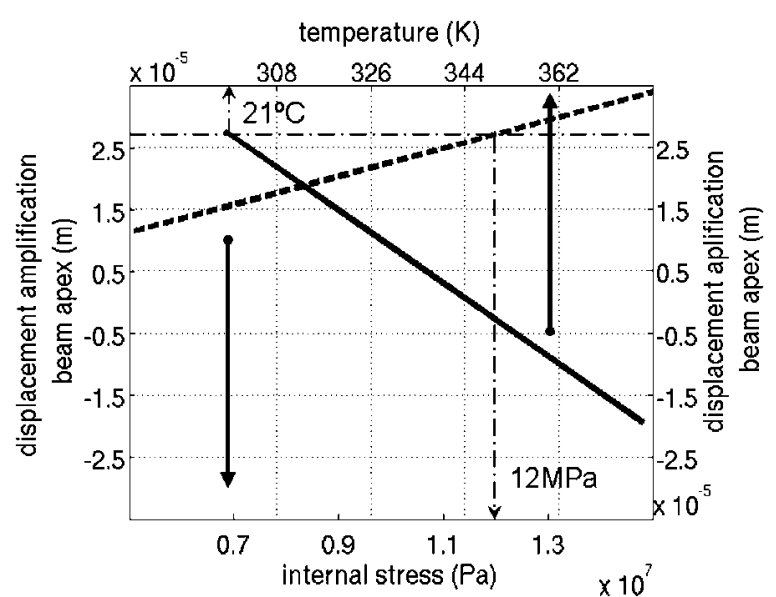

Figure 7. Simulated response showing a linear behavior of the displacement of the amplification beam to the internal stress (dashed line) and temperature (solid line). The dash-dot line is an example of a measured result: the vernier grating gives a response of $54 \mu \mathrm{m}$, resulting in a $27 \mu \mathrm{m}$ amplification beam displacement and this translates in $12 \mathrm{MPa}$ built-in stress at room temperature.

step is approximately 10 times higher than for SU-8. Thus, the indicator structures made of Epoclad can be headed using a conventional hot plate, and the stress indicator displacements, as a function of temperature, can be recorded using an optical microscope. A simulated example of the response of device 2 is displayed in figure 7.

\section{The fabrication process}

SU-8 was bought from Microchem. Corp., and LOR 10B was also bought from Microchem. Corp. as the sacrificial material for the fabrication process. Epoclad was bought from Micro Resist Technology GmbH.

To fabricate the free-standing structures, a surface micromachining technique was used. A sacrificial polyimide layer (LOR 10B) was spin coated on a 3 inch silicon wafer and patterned using a conventional lithography step. The SU-8 or
Epoclad is spin coated directly on to the patterned sacrificial layer. After a relaxing period, the epoxy film is soft baked on a leveled hot plate. For SU-8 the soft bake is done at $70{ }^{\circ} \mathrm{C}$ for $60 \mathrm{~min}$. For Epoclad the soft bake is done according to the datasheet provided by the manufacturer, except that the soft bake times were increased to avoid stiction to the mask during exposure. After the soft bake step the wafers were allowed to cool down and were exposed to $320 \mathrm{~mJ} \mathrm{~cm}^{-2}$ of UV light. Wavelengths below $360 \mathrm{~nm}$ were filtered out. To partially cure the photoepoxies, a post-exposure bake was done inside a conventional oven under a $\mathrm{N}_{2}$ atmosphere. Curing inside an oven helps to eliminate stress gradients because no major temperature gradients inside the film will exist compared to curing on a hot plate. For SU-8 the post-exposure bake step is done at $80{ }^{\circ} \mathrm{C}$ for $60 \mathrm{~min}$ and for Epoclad at $110{ }^{\circ} \mathrm{C}$ for $60 \mathrm{~min}$. The non-exposed epoxy film can be redissolved in PGMEA (propylene glycol monomethyl ether acetate) during a development step. A final hard bake step is also done in a conventional oven under a $\mathrm{N}_{2}$ atmosphere. For SU-8 this is done at $150{ }^{\circ} \mathrm{C}$ for $60 \mathrm{~min}$ and for Epoclad at $140{ }^{\circ} \mathrm{C}$ for $60 \mathrm{~min}$.

When the structures were fully cured after the hard bake step, the sacrificial layer is etched using an alkaline solution. The samples were then dried using a freeze drying step; see figure 8.

Depending on the curing time and temperature, the properties of SU-8 can vary significantly. This is no longer the case when the curing reaction saturates and the epoxy is fully cured [11]. The same behavior is also observed for Epoclad but not yet studied in depth because we wish to focus on the fully cured structure and the most stable condition of the polymer.

\section{Measurement details}

The displacement measurements were done using an optical microscope. The vernier grating at the amplification beam apex is used to record the exact displacement. This is shown in figure 9. For the stress measurements, approximately 

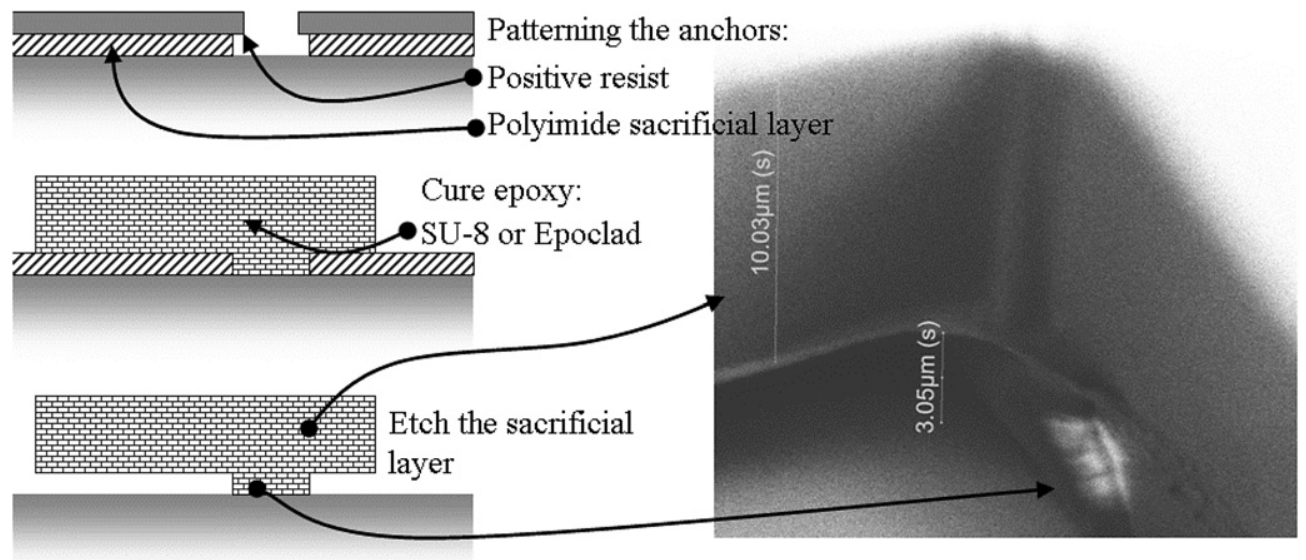

Figure 8. Fabrication process used to manufacture the stress indicator structures. The polyimide layer is patterned using a positive resist mask and etched in an alkaline solution. After removing the positive resist mask the epoxy is spin coated and patterned. Finally, the sacrificial layer is etched.

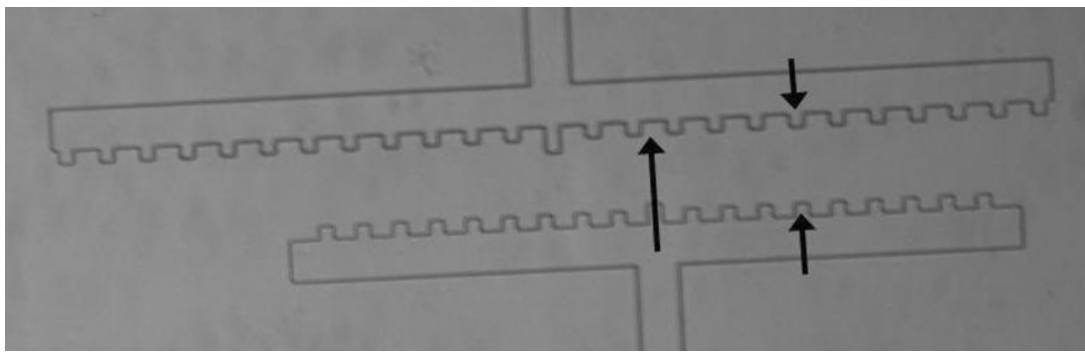

Figure 9. Photo by an optical microscope of a fabricated vernier grating on an indicator structure showing a displacement of $72 \mu \mathrm{m}$ resulting in an amplification beam displacement of $36 \mu \mathrm{m}$.

30 indicator structures per epoxy were evaluated and averaged.

It must be noted that the epoxies have a gel-like property. This means that they will absorb a significant amount of water or water vapors. As a result, the internal stress in the material will change as a function of humidity of the surrounding air $[11,15]$. Thus, the internal stress is defined as the stress measured at room temperature $\left(2{ }^{\circ} \mathrm{C}\right)$ and with a relative humidity of $54 \%$.

To determine the CTE, the samples were placed on a temperature-controlled hot plate. When a certain temperature was reached, $5 \mathrm{~min}$ of soak time was foreseen before the displacement of the indicator structures was recorded. This displacement was also recorded using an optical microscope and the vernier grating; see figure 10.

\section{Discussion}

Table 2 lists a summary of the obtained data for Epoclad. Data of SU-8 are also included for comparison. Calculating the stress in the material from the displacement indicated by the stress indicator structure is very straightforward. Using the simulation result of the previously discussed simulations, accurate conversion factors can be determined to convert the displacement of the vernier grating directly into stress. This is also possible because the response of the indicator structure is

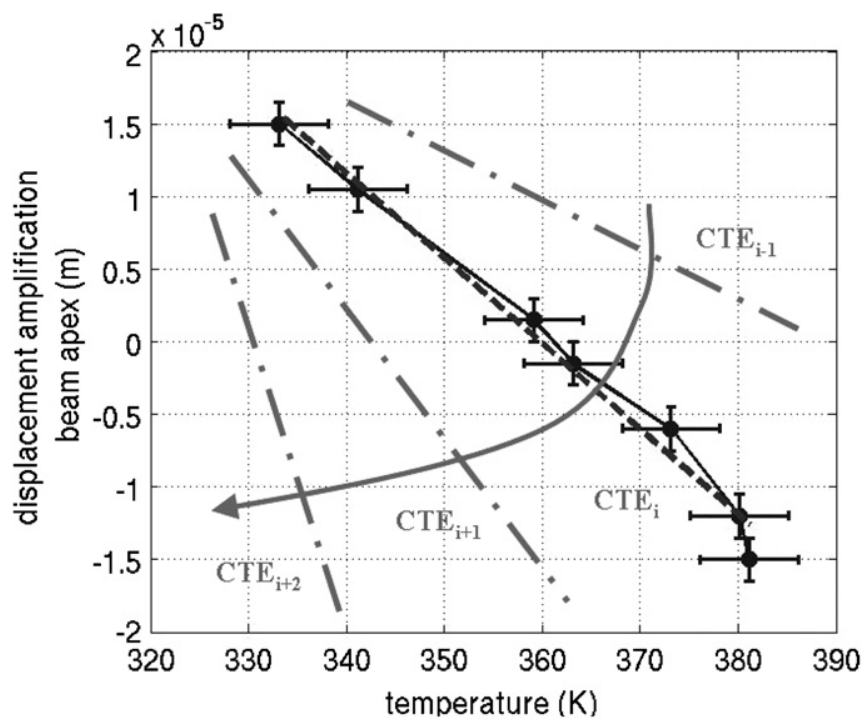

Figure 10. (a) Measured response of an indicator structure (device 2) fabricated in Epoclad. The vertical error bar is the indicator resolution and the horizontal error bar is $\pm 5^{\circ} \mathrm{C}$, as the upper limit of uncertainty on the temperature. The dashed line is a linear fit. (b) The dash-dot line is the response when the material would have a different CTE and illustrates the method used to determine the CTE.

very linear, as shown in figure 7 . The error in the conversion factors is very small compared to the spread of the indicator 
Table 2. Results of the measured in-plane internal stress and CTE of Epoclad.

\begin{tabular}{lll}
\hline & Epoclad & SU-8 2000 \\
\hline Measured internal stress $(\mathrm{MPa})$ & $12 \pm 2^{\mathrm{a}}$ & $18 \pm 4^{\mathrm{a}}$ \\
Reported internal stress $(\mathrm{MPa})$ & - & 15 [11], 30-40 [13] \\
Measured CTE $\left(\mathrm{ppm} \mathrm{K}^{-1}\right)$ & $87 \pm 10^{\mathrm{b}}$ & - \\
Reported CTE $\left(\mathrm{ppm} \mathrm{K}^{-1}\right)$ & - & $52[14]$ \\
\hline
\end{tabular}

${ }^{a}$ Standard deviation.

b $95 \%$ confidence bounds of linear fit.

structures. Thus, the error in the measured stresses is due to the spread of the approximately 30 indicator structures recorded.

For the determination of the CTE, a different approach is used in order to minimize the error in the calculation. From the measured graph, figure 10, the directional cosine of the linear fit is compared with the simulated data so that the response of the device lines up with the simulated response; this is also illustrated in figure 10.

The amplification beam apex displacement is a function of temperature and of the CTE $(\alpha)$. Doing a simulation will yield the following:

$$
d_{s}=f\left(\alpha_{i}, T_{i}\right) .
$$

Here $d$ is the simulated displacement, the $s$ in subscript indicates that this displacement is simulated and $\alpha_{i}$ and $T_{i}$ are the CTE and the temperature, respectively. Subscript $i$ indicates the discrete steps in the simulated data. Using the physical model of thermal expansion, with $\varepsilon=\alpha \cdot \Delta T$ and the fact that the indicator structures have a very linear response, the function $f$ has the following form and thus the directional cosine $\partial d / \partial T$ is linear in $\alpha$ :

$$
d=C_{1} \cdot \alpha \cdot \Delta T+C_{0} \quad \text { and thus } \quad \frac{\partial d}{\partial T}=\left(C_{1} \cdot \alpha\right) .
$$

Now, $C_{1}$ corresponds with the measured directional cosine from the graph in figure 10. Because the directional cosine is linear in $\alpha$, we can fit a straight line through the simulated $\partial d_{s} / \partial T$ and use it to find our CTE of Epoclad.

In other words, the CTE of Epoclad is calculated as follows:

$\alpha=\frac{\text { measured_directional_cosine }-\Phi}{\Psi}$ with $\Psi$ and $\Phi$

$$
\text { so that } \frac{\partial d_{s}}{\partial T}=\Psi \alpha+\Phi
$$

Because of the linearity of the structures, the fit parameters $\Psi$ and $\Phi$ have very small relative errors. Furthermore, $\Phi$ is negligible in comparison with the measured directional cosine. It will tend to zero for an ideal experiment. Hence, the CTE can be simply calculated and the error is of the same form as in the initial experiment, being the error of the linear fit in figure 10 .

The measured properties for SU-8 and Epoclad are very similar. This is not surprising because both of the materials are epoxies. Epoclad, however, has a lower internal stress than SU-8. This makes Epoclad a good epoxy to form thick layers. When a deposited layer has high internal stress, the adhesion force struggles to prevent the film from delamination, especially for thick films. Epoclad has good adhesion to silicon. But, whether this is the cause of higher adhesion forces and/or the cause of the lower internal stresses is still not clear. This will be a topic in the future work, to characterize the adhesion force itself.

\section{Conclusion}

The internal or built-in stress and the CTE of Epoclad are, according to the authors' knowledge for the first time, recorded. This is done using on-wafer stress indicator structures. SU-8, a well-reported material, is also investigated using the same indicator structures for comparison.

It is demonstrated that the CTE can be measured using on-wafer stress indicator structures when a polymer material is used.

Some important design considerations were explained for designing a polymer MEM structure using these high stress photoepoxy materials. The Epoclad resist is very well suited for this purpose. It can be used as an alternative to SU-8, where lower stresses and good adhesion are needed.

\section{Acknowledgments}

This work has been carried out in the frame of SBO-programme project 060046, Gemini, sponsored by the 'instituut voor de aanmoediging van Innovatie door Wetenschap en Technologie in Vlaanderen' (IWT), Belgium.

\section{References}

[1] Kato Y, Sekitani T, Takamiya M, Doi M, Asaka K, Sakurai T and Someya T 2007 Sheet-type braille displays by integrating organic field-effect transistors and polymeric actuators IEEE Trans. Electron Devices 54 202-9

[2] Engel J M, Chen J, Liu C and Bullen D 2006 Polyurethane rubber all-polymer artificial hair cell sensor J Microelectromech. Syst. 15 729-36

[3] Schroder $\mathrm{H}$ et al 2006 Waveguide and packaging technology for optical backplanes and hybrid electrical-optical circuit boards Proc. SPIE 6124 612407-14

[4] Zang T Y, Su Y J, Qian C F, Zhao M H and Chen L Q 2000 Microbridge testing of silicon nitride thin films deposited on silicon wafers Acta Mater. 48 2843-57

[5] Loy C T, Pradhan S C, Ng T Y and Lam K Y 1999 A series solution approach to an analytical load-deflection relation for the measurement of mechanical properties of thin films J. Micromech. Microeng. 9 341-4

[6] Gianchandani Y B and Najafi K 1996 Bent-beam strain sensors J. Microelectromech. Syst. 5 52-8

[7] Ericson F, Greek S, Soderkvist J and Schweitz J A 1995 High sensitive internal film stress measurement by an improved micromachined indicator structure Transducers 65-Eurosensors IX 84-7

[8] Elbrecht L, Storm U, Catanescu R and Binder J 1997 Comparison of stress measurement techniques in surface micromachining J. Micromech. Microeng. 7 151-4

[9] Goosen J F L, van Drieenhuizen B P, French P J and Wolffenbuttel R F 1993 Stress measurement structures for micromachined sensors Tech. Dig. 7th Int. Conf. On Solid State Sensors and Actuators, Transducers 93 783-6 
[10] Fried J R 2007 Polymer Science and Technology ed N Radhuber, B Goodwin and M Vicente (Upper Saddle River, NJ: Prentice-Hall)

[11] Feng R and Farris R J 2003 Influence of processing conditions on the thermal and mechanical properties of SU8 negative photoresist coatings J. Micromech. Microeng. 13 80-8

[12] Wouters K and Puers R 2008 Young's modulus measurement and creep behavior of three different photo definable epoxies for micro fabrication Eurosensor XXII pp 835-8
[13] Bachmann D, Schoberle B, Kuhne S, Leiner Y and Hierold C 2006 Fabrication and characterization of folded SU-8 suspensions for MEMS applications Sensors Actuators A 130-131 379-86

[14] SU-8 2000 series, datasheet at www.microchem.com

[15] Yamaue T and Doi M 2004 Theory of onedimensional swelling dynamics of polymer gels under mechanical constraint Phys. Rev. 69 041402-5 